TABLE DR1. SITE CHARACTERISTICS

\begin{tabular}{|c|c|c|c|c|c|}
\hline \multirow[t]{2}{*}{ Site } & \multirow[t]{2}{*}{ Location } & \multirow{2}{*}{$\begin{array}{c}\text { Elevation } \\
(\mathrm{m})\end{array}$} & \multirow{2}{*}{$\begin{array}{c}\text { Slope } \\
(\%)\end{array}$} & \multicolumn{2}{|c|}{ Rock fragment cover ${ }^{\dagger}$} \\
\hline & & & & DP (\%) & non-DP $(\%)$ \\
\hline $\mathrm{CV}$ & $\begin{array}{c}35^{\circ} 12^{\prime} 11^{\prime \prime} \mathrm{N} \\
115^{\circ} 52^{\prime} 09^{\prime \prime} \mathrm{W}\end{array}$ & 690 & 4 & 87 & 21 \\
\hline PB & $\begin{array}{c}33^{\circ} 51^{\prime} 51^{\prime \prime} \mathrm{N} \\
115^{\circ} 38^{\prime} 37^{\prime \prime} \mathrm{W}\end{array}$ & 610 & 5 & 91 & 40 \\
\hline FM & $\begin{array}{c}34^{\circ} 28^{\prime} 26^{\prime \prime} \mathrm{N} \\
116^{\circ} 42^{\prime} 09^{\prime \prime} \mathrm{W}\end{array}$ & $\begin{array}{l}990 \text { (FM1) } \\
950 \text { (FM2) }\end{array}$ & $\begin{array}{l}10 \text { (FM1) } \\
25 \text { (FM2) }\end{array}$ & 87 & 73 \\
\hline
\end{tabular}

${ }^{\ddagger} \mathrm{DP}=$ desert pavement 


\begin{tabular}{|c|c|c|c|c|c|c|c|c|c|c|c|c|}
\hline \multirow[t]{2}{*}{ Horizon } & \multicolumn{2}{|c|}{ Depth } & \multirow[t]{2}{*}{ Dry color ${ }^{+}$} & \multirow[t]{2}{*}{ Texture ${ }^{\S}$} & \multirow[t]{2}{*}{ Structure } & \multirow[t]{2}{*}{ Roots $^{\#}$} & \multirow[t]{2}{*}{ Pores $^{\dagger \dagger}$} & \multirow[t]{2}{*}{ Efferv. ${ }^{\text {* }}$} & \multirow[t]{2}{*}{$\mathrm{pH}$} & \multirow{2}{*}{$\begin{array}{c}\mathrm{EC} \\
\left(\mathrm{dS} \mathrm{m}^{-1}\right) \\
\end{array}$} & \multirow{2}{*}{$\begin{array}{c}\mathrm{Cl}^{-} \\
\left(\mathrm{mg} \mathrm{kg}^{-1}\right) \\
\end{array}$} & \multirow{2}{*}{$\begin{array}{r}\mathrm{NO}_{3}^{-}-\mathrm{N} \\
\left(\mathrm{mg} \mathrm{kg}^{-1}\right) \\
\end{array}$} \\
\hline & Description & Sampling $^{\dagger}$ & & & & & & & & & & \\
\hline \multicolumn{13}{|c|}{ Cima Volcanic Field, desert pavement (CV1): Typic Natrargid } \\
\hline$\overline{\text { Avk }}$ & $0-3$ & $0-10$ & $10 \mathrm{YR} 6 / 3$ & $\mathrm{cl}$ & $\begin{array}{l}\text { 3cocol, } \\
1 \mathrm{mpl} \\
2 \mathrm{msbk}\end{array}$ & $1 \mathrm{vf}$ & $3 \mathrm{mvs}$ & st & 9.1 & 1.0 & 77 & 8 \\
\hline $\mathrm{Bk}$ & $3-7$ & & $10 \mathrm{YR} 5 / 3$ & $\mathrm{cl}$ & $\begin{array}{c}3 \mathrm{cocol}, \\
2 \mathrm{~m}, 1 \mathrm{fabk}\end{array}$ & $1 \mathrm{vf}$ & $\begin{array}{l}1 \mathrm{ftu}, \\
2 \mathrm{vfvs}\end{array}$ & st & 9.1 & 1.0 & & \\
\hline Btk & $7-15.5$ & $10-20$ & $10 \mathrm{YR} 5 / 3$ & 1 & $\begin{array}{c}3 \mathrm{vf}, \mathrm{f}, \mathrm{m} \\
\mathrm{abk}\end{array}$ & $1 \mathrm{vf}$ & $1 \mathrm{vfvs}$ & ne & 8.8 & 5.2 & 384 & 61 \\
\hline Btky & $15.5-27$ & $20-30$ & 7.5 YR $5 / 3$ & $\mathrm{cl}$ & $\begin{array}{c}2 \mathrm{vf}, 1 \mathrm{co} \\
\mathrm{sbk}\end{array}$ & $1 \mathrm{vf}$ & $1 \mathrm{fvs}$ & ne & 8.3 & 12.8 & 2496 & 207 \\
\hline Btkyz & $27-46$ & $30-40$ & 7.5 YR $5 / 3$ & $\mathrm{scl}$ & $2 f$,msbk & & $\begin{array}{l}2 \mathrm{fvs}, \\
2 \mathrm{vftu}\end{array}$ & ne & 7.7 & 13.1 & 1905 & 182 \\
\hline \multirow[t]{3}{*}{ Bkyzl } & $46-75$ & $40-50$ & 7.5 YR $5 / 4$ & $\mathrm{scl}$ & $2 \mathrm{f}, \mathrm{msbk}$ & & $2 \mathrm{vftu}$ & sl & 7.9 & 11.4 & 240 & 15 \\
\hline & & $50-60$ & & & & & & & & & 160 & 9 \\
\hline & & $60-70$ & & & & & & & & & 102 & 4 \\
\hline \multirow[t]{3}{*}{ Bkyz2 } & $75-100$ & $70-80$ & $7.5 \mathrm{YR} 5 / 3$ & scl & $3 \mathrm{f}, \mathrm{msbk}$ & & & ve & 8.0 & 9.3 & 315 & 15 \\
\hline & & $80-90$ & & & & & & & & & 468 & 29 \\
\hline & & $90-100$ & & & & & & & & & 1460 & 123 \\
\hline \multicolumn{13}{|c|}{ Cima Volcanic Field, non-pavement (CV2): Typic Paleargid } \\
\hline$\overline{\mathrm{A}}$ & $0-1$ & $0-4$ & $10 \mathrm{YR} 6 / 3$ & sl & 2f,m,copl & $2 v f$ & $2 v f t u$ & ne & 8.3 & 0.2 & 86 & 11 \\
\hline Bwl & $1-4$ & & $10 \mathrm{YR} 5 / 3$ & sl & $\begin{array}{c}2 \mathrm{vf}, 1 \mathrm{~m} \\
\mathrm{sbk}\end{array}$ & $2 \mathrm{vf}$ & $2 v f v s$ & ne & 8.3 & 0.2 & & \\
\hline Bw2 & $4-11$ & $4-10$ & $10 Y R 6 / 4$ & $\mathrm{sl}$ & $\begin{array}{l}2 \text { copl, } \\
1 \text { fabk, } \\
1 \mathrm{vfsbk}\end{array}$ & $2 \mathrm{vf}$ & $1 \mathrm{vfvs}$ & ne & 8.2 & 0.1 & 47 & 7 \\
\hline $\mathrm{Bt}$ & $11-22$ & $10-20$ & $7.5 \mathrm{YR} 4 / 6$ & $\mathrm{scl}$ & $\begin{array}{c}2 \mathrm{vff,f,m}, \\
\text { cosbk }\end{array}$ & $1 \mathrm{vf}$ & $1 \mathrm{vf}, \mathrm{ftu}$ & ne & 7.8 & 0.1 & 107 & 3 \\
\hline Btc & $22-31$ & $20-30$ & $7.5 \mathrm{YR} 4 / 6$ & $\mathrm{scl}$ & $\begin{array}{c}3 \mathrm{fpr} \\
3 \mathrm{fabk}\end{array}$ & $1 \mathrm{vf}$ & $\begin{array}{l}2 \mathrm{vf} \\
1 \mathrm{mtu}\end{array}$ & ne & 8.5 & 0.2 & 148 & 9 \\
\hline \multirow[t]{2}{*}{ Btck } & $31-58$ & $30-40$ & 7.5 YR 5/6 & $\mathrm{scl}$ & $\begin{array}{c}\text { 3mpr, } \\
\text { 3f,mabk }\end{array}$ & $\begin{array}{c}2 \mathrm{vf}, \\
1 \mathrm{f}, \mathrm{m}, \mathrm{co}\end{array}$ & $\begin{array}{c}2 \mathrm{vf}, 1 \mathrm{f}, \\
\text { cotu }\end{array}$ & ne & 8.5 & 0.2 & 132 & 5 \\
\hline & & $40-50$ & & & & & & & & & 74 & 8 \\
\hline \multirow[t]{5}{*}{ Btk } & $58-100$ & $50-60$ & 7.5 YR $4 / 6$ & $\mathrm{c}$ & $2 \mathrm{vf}$, fabk & $1 \mathrm{vf}, \mathrm{f}, \mathrm{m}$ & $\begin{array}{c}2 \mathrm{vfvs}, \\
2 \mathrm{vf}, 1 \mathrm{co} \\
\text { tu }\end{array}$ & ve & 8.3 & 0.3 & 84 & 9 \\
\hline & & $60-70$ & & & & & & & & & 77 & 11 \\
\hline & & $70-80$ & & & & & & & & & 67 & 10 \\
\hline & & $80-90$ & & & & & & & & & 79 & 10 \\
\hline & & $90-100$ & & & & & & & & & 84 & 10 \\
\hline
\end{tabular}




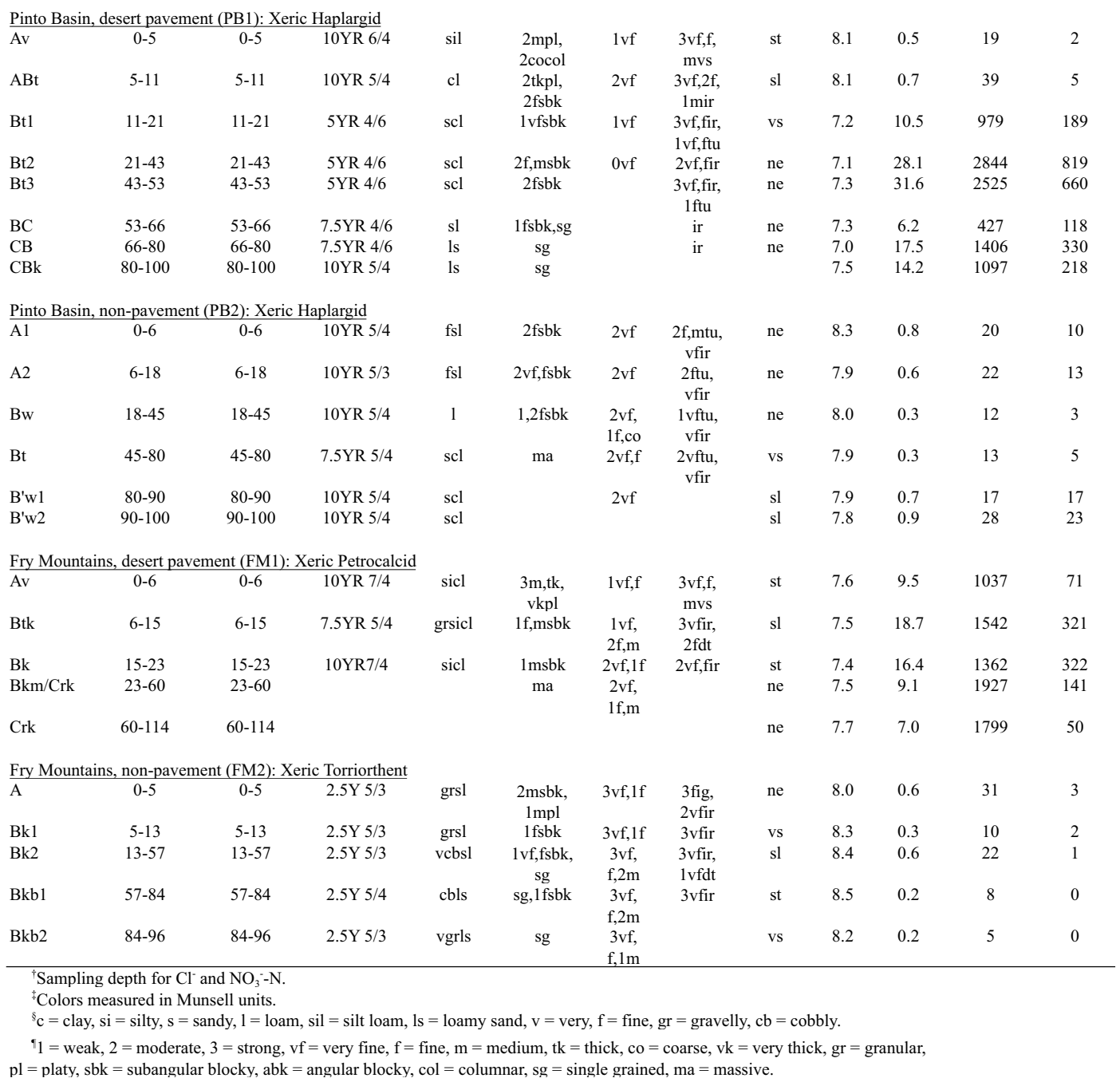


${ }^{\sharp} 0=$ very few, $1=$ few, $2=$ common, $3=$ many, $\mathrm{v}=$ very, $\mathrm{f}=$ fine, $\mathrm{m}=$ medium, $\mathrm{co}=$ coarse.

${ }^{\dagger} 1$ = few, 2 = common, 3 = many, $\mathrm{vf}=$ very fine, $\mathrm{f}=$ fine, $\mathrm{m}=$ medium, $\mathrm{vs}=$ vesicular, tu = tubular, ir $=$ interstitial, $\mathrm{dt}=\mathrm{dendritic}$ tubular, ig $=$ irregular. ${ }^{\mathrm{ft}}$ Effervescence class; $\mathrm{ne}$ = noneffervescent, vs = very slightly effervescent, $\mathrm{sl}$ = slightly effervescent, $\mathrm{st}$ = strongly effervescent, $\mathrm{ve}=$ violently effervescent. 
DR2008062

\section{References for analytical methods}

The fine earth fraction was extracted with deionized water and the leachates were analyzed for $\mathrm{Cl}^{-}$by automated coulometric titration (Frankenberger et al., 1996) and $\mathrm{NO}_{3}{ }^{-}$ colorimetrically using an autoanalyzer (Mulvaney, 1996).

Frankenberger, W.T., Tabatabai, M.A., Adriano, D.C., and Doner, H.E., 1996, Bromine, chlorine, and fluorine, in Sparks, D.L., ed., Methods of soil analysis, Part 3: Madison, WI, Soil Science Society of America, p. 833-868.

Mulvaney, R.L., 1996, Nitrogen - inorganic forms, in Sparks, D.L., ed., Methods of soil analysis, Part 3: Madison, WI, Soil Science Society of America, p. 1123-1184. 